\title{
The MOONRISE: payload for mobile selective laser melting of lunar regolith
}

Jörg Neumann, Mathias Ernst, Patrick Taschner, Niklas Gerdes, Simon Stapperfend, et al.

Jörg Neumann, Mathias Ernst, Patrick Taschner, Niklas Gerdes, Simon Stapperfend, Stefan Linke, Christoph Lotz, Jürgen Koch, Peter Wessels, Enrico Stoll, Ludger Overmeyer, "The MOONRISE: payload for mobile selective laser melting of lunar regolith," Proc. SPIE 11852, International Conference on Space Optics - ICSO 2020, 118526T (11 June 2021); doi: $10.1117 / 12.2600322$

SPIE Event: International Conference on Space Optics - ICSO 2021, 2021, Online Only 


\section{International Conference on Space Optics-ICSO 2020}

Virtual Conference

30 March-2 April 2021

Edited by Bruno Cugny, Zoran Sodnik, and Nikos Karafolas
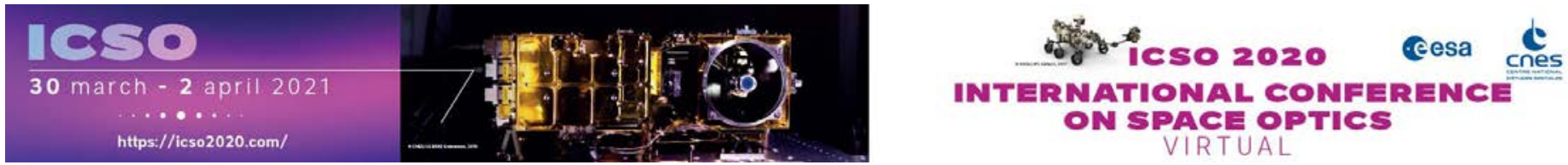

\section{The MOONRISE: payload for mobile selective laser melting of lunar regolith}

\section{Cesa isoporeseatings denes}




\title{
The MOONRISE-Payload for Mobile Selective Laser Melting of Lunar Regolith
}

\author{
Jörg Neumann*a, Mathias Ernst ${ }^{\mathrm{a}}$, Patrick Taschner ${ }^{\mathrm{a}}$, Niklas Gerdes ${ }^{\mathrm{a}}$, Simon Stapperfend ${ }^{\mathrm{b}}$, Stefan \\ Linke $^{\mathrm{b}}$, Christoph Lotz ${ }^{\mathrm{c}, \mathrm{d}}$, Jürgen Koch ${ }^{\mathrm{a}}$, Peter Wessels ${ }^{\mathrm{a}}$, Enrico Stoll ${ }^{\mathrm{b}}$, Ludger Overmeyer ${ }^{\mathrm{a}, \mathrm{c}}$ \\ ${ }^{a}$ Laser Zentrum Hannover e.V., Hollerithallee 8, D-30419 Hannover; ${ }^{b}$ Institute of Space Systems, \\ Technische Universität Braunschweig, Hermann-Blenk-Str. 23, D-38108 Braunschweig; ' Institute of \\ Transport and Automation Technology, Leibniz University Hannover, An der Universität 2,

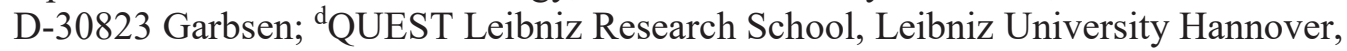 \\ Callinstraße 36, D-30167 Hannover
}

\begin{abstract}
In-Situ Resource Utilization (ISRU) technologies pave the way for a sustainable colony on the Moon. Above all, the construction of structures using only the available resources is an important factor in reducing costs and logistical effort. The MOONRISE project aims to melt lunar regolith using lasers on mobile platforms for the additive manufacturing of structures. This process is called Mobile Selective Laser Melting (M-SLM) and has the advantage that only electrical energy and a moving system are required. For a proof-of-principle experiment of M-SLM, which aims for creating 0D, 1D and 2D fused regolith structures on the lunar surface, we designed the MOONRISE payload. The MOONRISE payload can be accommodated on a rover or a robotic arm to ensure mobility for the melting experiments.

An Engineering Model (EM) of the payload including a fiber coupled diode laser was developed and environmentally tested. The dimension of the payload is $1.5 \mathrm{U}$ CubeSat. It has a mass of about $2.5 \mathrm{~kg}$ with further reduction potential towards flight model (FM) development. Verification tests with the EM were continued by attaching it to a robotic arm to create $2 \mathrm{D}$ regolith structures, i.e. flat rectangular specimens. Further tests with the EM were carried out under low gravity conditions in the large-scale research device Einstein-Elevator at the Hannover Institute of Technology (HITec), which allows experiments under zero gravity conditions for about four seconds. The Einstein-Elevator also enables adjustment of the gravity level from 0 to $5 \mathrm{~g}$, a feature that was used to carry out melting experiments with the EM under lunar gravity conditions.
\end{abstract}

Keywords: ISRU, laser melting, additive manufacturing, sintering, lunar exploration, construction

\section{INTRODUCTION}

When setting up a lunar station, technologies for the use of locally available materials are crucial. Such technologies drastically reduce the need for transportation from Earth. We aim to provide proof of a key technology, namely Mobile Selective Laser Melting (M-SLM) for terrain modelling i.e. for building large structures such as launch/landing pads and streets, but also building infrastructures such as shelters protecting astronauts or equipment against radiation and micrometeorites on the Moon. Based on an internal trade-off between various methods for additive manufacturing of regolith we think that using a laser is the best option, since it is independent from the sun and enables good precision as well as the potential for building large structures. M-SLM can be fully automated. For M-SLM, a mobile high power laser beam is directly directed on lunar regolith leading to its melting and after cooling to solid structures.

*j.neumann@1zh.de; phone +49 5112788 210; fax +49 5112788 100; www.lzh.de 
Laser-based processing tests of regolith and simulants have been performed so far solely in laboratories on Earth [1]. The underlying system technology for processes such as $\mathrm{SLM}^{\circledR}$ is complex. Structures are created layer by layer, which requires advanced powder processing and distribution system technology, which would in turn require large investments when being realized for application on the lunar surface. This can only be considered as a long-term goal as shown in the roadmap of Fig. 1. Within any type of laboratory experiments on Earth, it is not possible to fully simulate the lunar environment, resulting in potential unrealistic processes. In addition, unknown effects can emerge on-site, which are completely unpredictable and which may have a big impact on processing results. With that lack of knowledge, it is not possible to plan realistic missions for the construction of infrastructures.
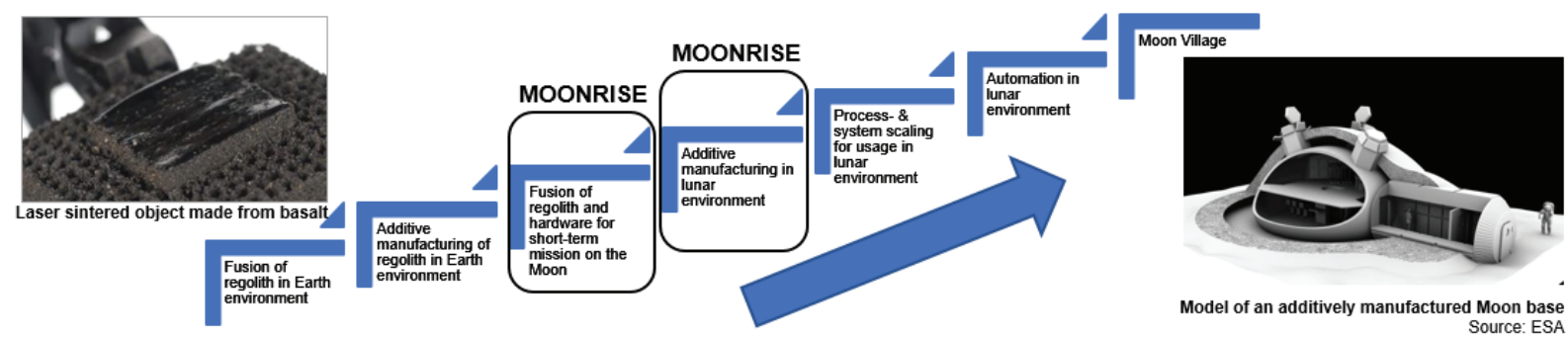

Figure 1. Roadmap for additive ISRU construction on the Moon.

Therefore, the two partners, Institute of Space Systems (IRAS) and Laser Zentrum Hannover e.V. (LZH), teamed up for the development of the MOONRISE payload, which should serve as a proof-of-principle experiment for M-SLM on the lunar surface. The MOONRISE payload is currently at post-PDR-status with a tested Engineering Model (EM) available. It consists of a high power laser capable of melting regolith at a fixed spot, i.e. creating 0D objects from fused regolith. However, by accommodating the payload on a rover or a robotic arm 1D and 2D objects could be created by the added mobility. The MOONRISE payload could enable a timely and inexpensive test of laser melting of regolith on the lunar surface. This direct proof of feasibility would significantly increase the technological readiness level of the M-SLM technology. It is expected that the additive processing of regolith will then be increasingly taken into account in concepts for exploring the Moon.

\section{ENGINEERING MODEL DEVELOPMENT AND TESTING}

Synthetic lunar soils, so-called regolith simulants, are typically used for experiments on Earth due to a lack of original material from the Moon. This enables realistic results, even if it is not possible to completely reproduce all the properties of the lunar soil and the prevailing environmental conditions. For the initial laboratory experiments, we used different variants of regolith, which were produced with the help of a modular regolith simulant system developed at IRAS [2]. We placed the regolith simulants in a vacuum chamber at around $10^{-2} \mathrm{mbar}$. The regolith simulant was irradiated for the melting experiments by an external diode laser at a wavelength of $976 \mathrm{~nm}$ and a power of up to $140 \mathrm{~W}$ for several seconds. Melting was observed in the distinct optimized optical setup to start at $35 \mathrm{~W}$ of optical power for less than $10 \mathrm{~s}$. Spherical beads from melted regolith with a diameter of a few millimeter were produced (Fig. 2).
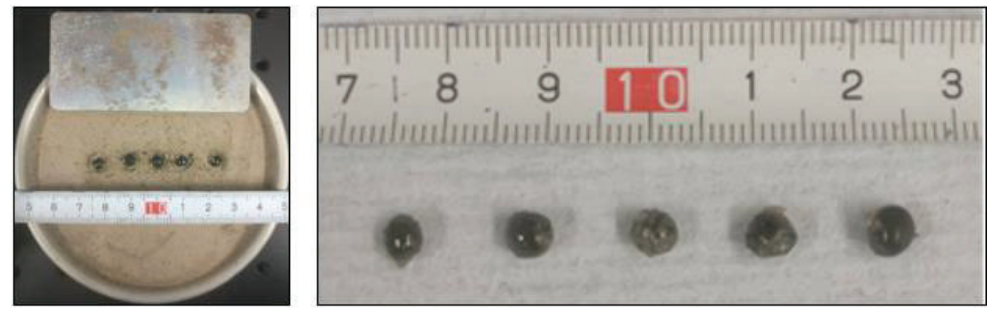

Figure 2. Laser melted regolith simulant spheres. 
Moreover, it could be observed that a working distance of $25 \mathrm{~cm}$ from the exit optics is safe to protect the optics from contamination induced by the laser regolith interaction. With this laboratory setup, major design parameters for the payload such as beam guiding, working distance, spot size and optical power range could be deduced. The MOONRISE payload mainly consists of a printed circuit board (PCB) for system communication, a fiber coupled diode laser, an electrical diode driver, a beam focusing optics, and an LED illumination as depicted in the block diagram (Fig. 3). The visualization of the molten regolith structures will be obtained by external cameras of the rover or the lander.

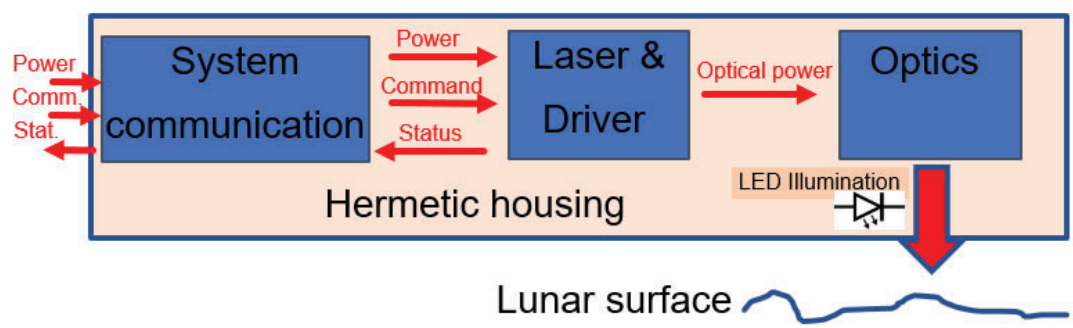

Figure 3. Block diagram of the MOONRISE payload.

For reasons of cost-efficiency, the Engineering Model (EM) of the MOONRISE payload was developed based on a highrisk approach by means of mainly using commercial off-the-shelf (CotS) components. These CotS parts were partly based on space heritage and have been screened in environmental tests and selected before. Also for the EM, a diode laser system with an output power of up to $140 \mathrm{~W}$ at a wavelength of $976 \mathrm{~nm}$ was chosen. Nevertheless, the package, beam shaping, and main communication and control electronic (PCB) are fully custom made for this payload to meet the mission requirements. Custom PCB components meet the Automotive Electronics Council (AEC) qualification requirements [3]. Within this qualification, they passed load tests for high temperature operating life (HTOL) grade 1 which means they are proven to work within the temperature range from $-40^{\circ} \mathrm{C}$ to $125^{\circ} \mathrm{C}$. The laser system is designed for a working distance of $250 \mathrm{~mm}$ between the laser output and the regolith surface but is capable to conduct the melting process efficiently with a deviation of $\pm 30 \mathrm{~mm}$. Even if the payload mission requires only operation times of few seconds, the system is capable to operate up to several minutes at once and stay within its thermal boundaries without any active thermal control. To reduce the risk of failure of the used CotS components, the package is hermetically sealed and filled with dry air with low dew point to prevent condensation during cruise and surface mission. A lightweight version of this package is currently under development to reduce the mass of the payload to well below $2 \mathrm{~kg}$. For baseline operation, a laser power of typically $70 \mathrm{~W}$ will be applied for $6 \mathrm{~s}$ to the lunar surface at a distance of about $25 \mathrm{~cm}$. The LED illumination supports visualization of the molten regolith by external cameras. The MOONRISE payload can be accommodated on a rover or a robotic arm to ensure some mobility for the melting experiments. The EM, which is shown with its major specifications in Fig. 4, has been assembled and tested.

\begin{tabular}{|l|c|}
\hline Parameter & MOONRISE \\
\hline $\begin{array}{l}\text { Optical output power } \\
\text { capabilities }\end{array}$ & $\begin{array}{c}6-140 \mathrm{~W} \\
\text { typ. } 70 \mathrm{~W}\end{array}$ \\
\hline Power consumption (laser on) & $\begin{array}{c}25 \mathrm{~W}-340 \mathrm{~W} \\
\text { typ. } 175 \mathrm{~W} \text { for } 6 \mathrm{~s}\end{array}$ \\
\hline Mass & $\begin{array}{c}\sim 25 \mathrm{~kg} \\
\text { (further reduction of } 750 \mathrm{~g} \text { ) }\end{array}$ \\
\hline Dimensions & $1.5 \mathrm{U}$ \\
\hline Distance to ground & $250+/-30 \mathrm{~mm}$ \\
\hline Operating temperature & $-35^{\circ} \mathrm{C}$ to $+70^{\circ} \mathrm{C}$ (tbc) \\
\hline Storage temperature & $-50^{\circ} \mathrm{C}$ to $+95^{\circ} \mathrm{C}$ (tbc) \\
\hline
\end{tabular}
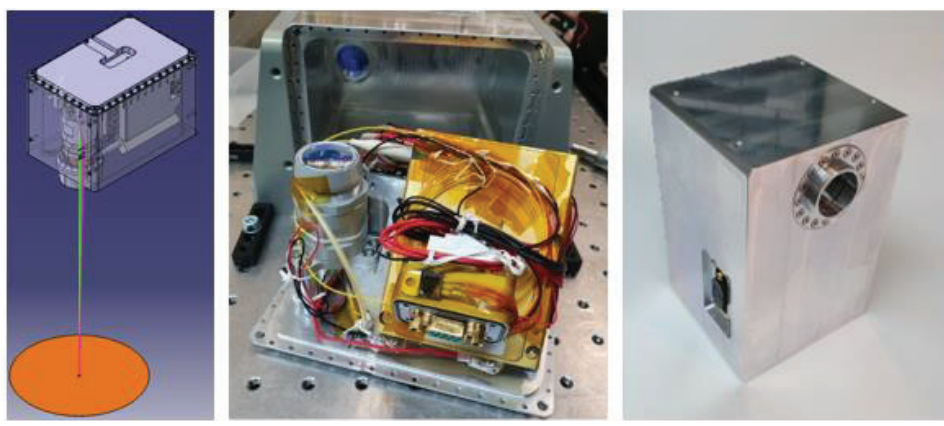

Figure 4. MOONRISE EM: Major specification table, CAD model with laser beam, open EM, hermetically sealed EM package. 
At first, functional tests with the MOONRISE EM attached to a vacuum chamber have been carried out. The melting and solidification of regolith were comparable to the initial laboratory experiments and are depicted in Fig. 5.
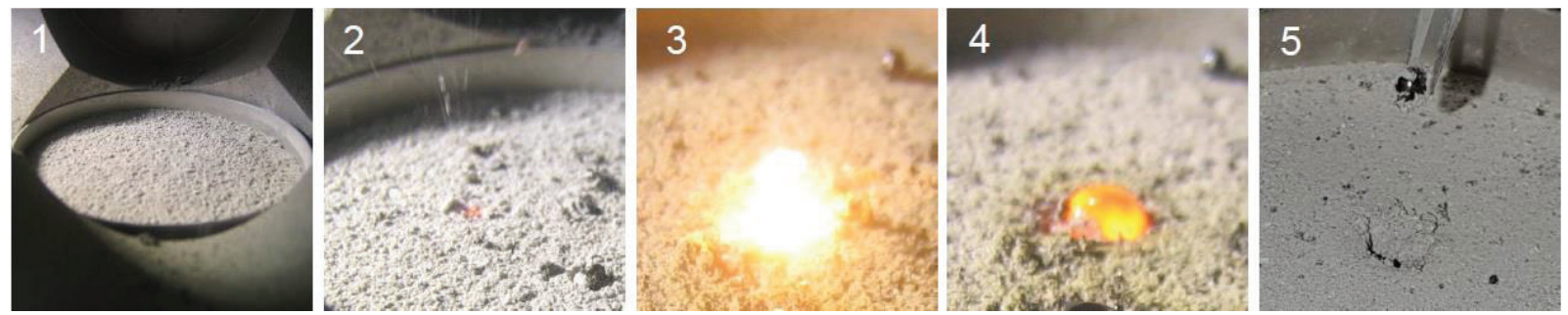

Figure 5. Regolith melting process with the MOONRISE EM: 1. Sample container with regolith simulant, 2. Start of the melting process: some simulant particles are ejected, 3. Melting phase: a sphere of molten simulants forms, 4 . Cooling phase: the molten regolith solidifies amorphously, 5. Cooled and solidified sample.

The performance, i.e. optical output power of the MOONRISE EM at different temperatures was evaluated in a thermal vacuum test campaign (Fig. 6 left). The non-operating temperatures ranging from $-50{ }^{\circ} \mathrm{C}$ to $95{ }^{\circ} \mathrm{C}$ did not lead to any damage or degradation of the payload. At low temperatures, the laser was able to produce more output power than expected in forehand. At the minimum operating temperature of $-35{ }^{\circ} \mathrm{C}$, for example, the output power was $15 \%$ higher than at room temperature. Between $30^{\circ} \mathrm{C}$ and $50^{\circ} \mathrm{C}$, the performance was good enough to fulfill all mission requirements. Up to $70^{\circ} \mathrm{C}$, the performance was sufficient for the minimum mission goal, which is the production of one spherical sample. The thermal-vacuum qualification campaign did not lead to any permanent degradation of the MOONRISE EM.

Sine and random vibration tests were performed for full qualification level (Fig. 6 right). This includes sine vibration up to $10 \mathrm{~g}$ with a frequency up to $100 \mathrm{~Hz}$ and random vibration with $16.31 \mathrm{~g}_{\text {rms }}$ in the range between $20 \mathrm{~Hz}$ and $2 \mathrm{kHz}$. These tests revealed a minor issue within the diode driver and thus showed the need to customize it further. The diode laser, the in-house-developed PCB, mechanics and all other optical elements passed all tests without any degradation or failure.
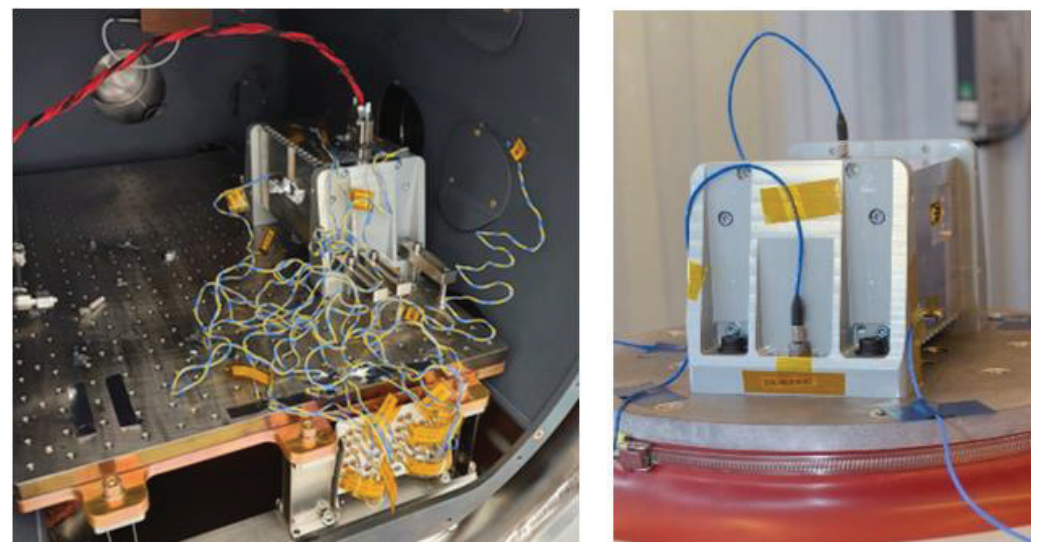

Figure 6. Environmental testing: MOONRISE EM in thermal vacuum chamber (left) and on vibration table (right). 


\section{FURTHER PAYLOAD VERIFICATION}

\subsection{Lunar gravity condition}

For further verification, we obtained the unique opportunity to use the novel Einstein-Elevator at the Hannover Institute of Technology (HITec), University of Hannover [4-6]. The Einstein-Elevator is an active drop tower, which enables experiments to be performed under different gravity conditions. We integrated the MOONRISE EM setup (Fig. 7 right) into the Einstein-Elevator experiment carrier (Fig. 7 left).

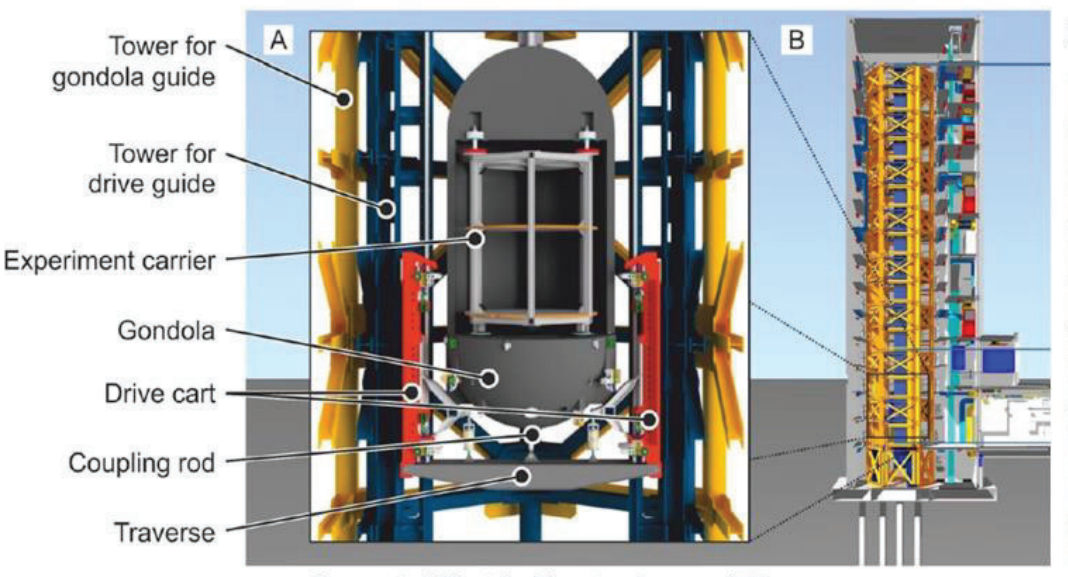

Concept of Einstein-Elevator (source: ITA)

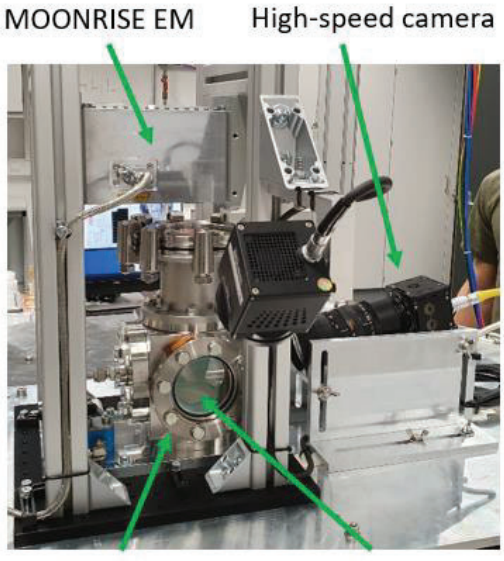

Vacuum chamber Regolith sample

Figure 7. Schematic drawing of the Einstein-Elevator (left) and laser melting experiment prepared for the experiment carrier (right).

Several laser melting experiments of regolith simulants could be executed in two measurement campaigns not only under micro-gravity $(\approx 0 g)$, but also under lunar gravity conditions $(0.16 g)$ and compared to the experiments under Earth gravity. The tests were carried out under all three gravity conditions with an identical setup and laser parameters. The irradiation time was $3 \mathrm{~s}$ due to the limited drop tower height, the applied optical power was therefore increased to $105 \mathrm{~W}$. All received samples have a spherical shape and a diameter of approximately $3 \mathrm{~mm}$. The surface is glass-like and regolith particles are fused to the underside of the regolith spheres. Minor differences were found between the experiments under different gravity conditions and are described elsewhere in detail [7]. However, the process of melting regolith beads could be proven.
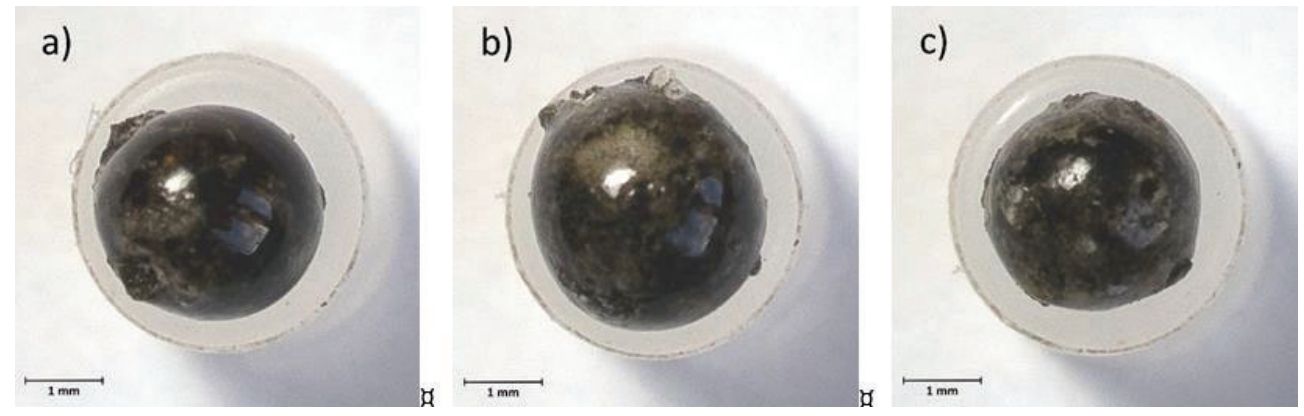

Figure 8. Typical samples of molten regolith, a) produced at $1 \mathrm{~g}$, b) at $0.16 \mathrm{~g}$ and c) at $0 \mathrm{~g}$ gravity condition. 


\subsection{Accommodation on a robotic arm}

In addition, we attached the MOONRISE EM to a robotic arm ([8], Fig. 9 left). The goal of the experiment was to test the M-SLM process for the MOONRISE payload on a robotic arm for identification of parameters to produce flat rectangular specimens. This lays the knowledge foundation for the production of flat infrastructure on the lunar surface such as streets or landing pads. On the other hand, it is an important step for the production of three-dimensional objects using the M-SLM process. The experiment was carried out under Earth conditions, such as Earth gravity, Earth atmosphere and room temperature. The laser was positioned by the robotic arm so that its distance to the regolith simulant surface was constantly $25 \mathrm{~cm}$. The laser power was set to the maximum value of $120 \mathrm{~W}$ and the driving velocity of the laser was set to $1 \mathrm{~mm} / \mathrm{s}$ by moving the robotic arm. With these parameters several lines were fused together and a solid 2D structure of $20 \mathrm{~mm} \times 20 \mathrm{~mm} \times 4 \mathrm{~mm}$ in size was generated (Fig. 9 right). The produced specimens had a mass of a few grams and varied in their mass by only a few percent. The method is reproducible despite the inhomogeneous simulant material that was processed. The samples had an amorphous composition with some embedded crystalline particles on the contact side with the simulant bed. The contact points of the individual lines molten by the laser are stable, which was tested by applying some load manually. Thus, it can be assumed that larger components can be manufactured with the process.
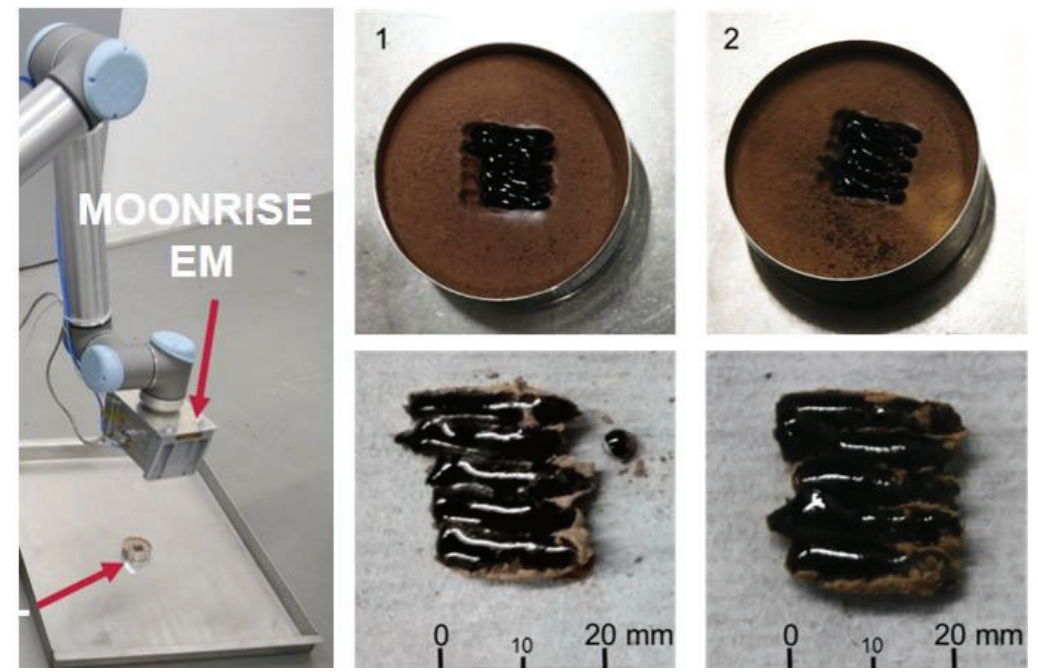

Figure 9. Typical samples of molten 2D-regolith structures (right) generated by attaching the MOONRISE EM to a robotic $\operatorname{arm}($ left).

\section{SUMMARY}

In summary, an Engineering Model (EM) of the MOONRISE payload for a proof-of-concept experiment for M-SLM on the lunar surface was developed and environmentally tested. The dimension of the payload is $1.5 \mathrm{U}$ CubeSat. It has a mass of about $2.5 \mathrm{~kg}$ with further reduction potential towards flight model (FM) development. The process of M-SLM process was verified in vacuum in the Einstein-Elevator under lunar gravity conditions producing melted regolith spheres with a diameter of $\approx 3 \mathrm{~mm}$ by fixed spot irradiation. For verification of the procedure for generation of larger structures, the EM was attached to a robotic arm. By moving this robotic arm, flat rectangular specimens with a size of $20 \mathrm{~mm} x$ $20 \mathrm{~mm}$ x $4 \mathrm{~mm}$ could be generated. 


\section{ACKKNOWLEDGEMENT}

We would like to thank the VolkswagenStiftung for funding the MOONRISE project within the scope of the open - for the unusual ("Offen - für Außergewöhnliches") program (Az. 94647 \& Az. 94890).

\section{REFERENCES}

[1] N. Gerdes, L. G. Fokken, S. Linke, S. Kaierle, O. Suttmann, J. Hermsdorf, E. Stoll, C. Trentlage, "Selective Laser Melting for processing of regolith in support of a lunar base," J. Laser Appl. 30, 032018 (2018).

[2] S. Linke, L. Windisch, N. Kueter et al., "TUBS-M and TUBS-T based modular Regolith Simulant System for the support of lunar ISRU Activities," Planet Space Sci 180, 104747 (2020).

[3] AEC Component Technical Committee. AEC documents. n.d..http://www.aecouncil.com/AECDocuments.html. Last retrieved on 24 Nov 2020.

[4] C. Lotz, T. Froböse, A. Wanner, L. Overmeyer, W. Ertmer, "Einstein-Elevator: A New Facility for Research from $\mu g$ to $5 g$," Grav. Space Res., 5(2), 11-27 (2017).

[5] C. Lotz, N. Gerdes, R. Sperling, S. Lazar, S. Linke, J. Neumann, E. Stoll, W. Ertmer, L. Overmeyer, "Tests of additive manufacturing and other processes under space gravity conditions in the Einstein-Elevator," Logistics Journal, Vol. 2020, 1-12, DOI: 10.2195/lj_Proc_lotz_de_201310_01 (2020).

[6] C. Lotz, Y. Wessarges, J. Hermsdorf, W. Ertmer, L. Overmeyer, "Novel active driven drop tower facility for microgravity experiments investigating production technologies on the example of substrate-free additive manufacturing," Adv. Space Res. 61(8), 1967-1974 (2018).

[7] S. Stapperfend, N. Gerdes, S. Linke, M. Ernst, P. Taschner, J. Koch, P. Wessels, J. Neumann, E. Stoll, L. Overmeyer, "Laser Melting of Lunar Regolith Simulant under Different Gravity Conditions Using the Moonrise-Payload," $8^{\text {th }}$ European Lunar Symposium, Virtual Workshop May 12-14, 2020, https://els2020.arc.nasa.gov/playback, https://www.youtube.com/watch?v=dyYQITUpXu0

[8] A. Voß, R. Freund, S. Harms, S. Linke, E. Stoll, "MIRA3D - a terrestrial robotic prototype for mobile additive layer manufacturing of lunar regolith," IAC-18-A3.2B.11, 43642 (2018). 